\title{
AVALIAÇÃO DA POSTURA EM CRIANÇAS DO ENSINO FUNDAMENTAL: ATITUDES E HÁBITOS CORPORAIS POSITIVOS E ALTA PREVALÊNCIA DE ALTERAÇÕES POSTURAIS
}

Maressa Lopes Menezes, Júlia Maia Viudes Agostinho, Dyenifer Fernandes de Oliveira, Nathália Serafim da Silva, Ana Lúcia de Jesus Almeida, Renilton José Pizzol

Universidade Estadual Paulista - UNESP, Departamento de Fisioterapia, Presidente Prudente, SP. E-mail: maressa.mezes@gmail.com

\section{RESUMO}

A escola é considerada um espaço potencial para o desenvolvimento do cuidado à saúde. O Fisioterapeuta pode participar de ações de saúde no contexto em questão, principalmente em adoção de hábitos posturais, iniciando com a avaliação de alterações posturais. Este estudo objetivou a avaliação postural de escolares de Ensino Fundamental do município de Presidente Prudente. A metodologia constou da elaboração de Ficha de Avaliação Postural contendo dados individuais; questionários de hábitos corporais e atitudes posturais e avaliação postural estática. Os resultados foram 42 crianças avaliadas; idade média de $9,8 \pm 0,9$ anos; grupo ativo fisicamente; boa percepção postural durante as atividades cotidianas e alta prevalência de alterações posturais, principalmente nos segmentos cabeça, ombros e coluna lombar. Concluímos que escolares de Ensino Fundamental possuem bons hábitos corporais e posturais, além de grande prevalência de algias e alterações posturais. Esses aspectos reforçam a necessidade de intervenção fisioterapêutica precoce.

Palavras-chave: serviços de saúde escolar, fisioterapia, postura, escolares, ensino fundamental

\section{EVALUATION OF POSTURE IN CHILDREN OF FUNDAMENTAL TEACHING: ATTITUDES AND BODY HABITS POSITIVE AND HIGH PREVALENCE OF POSTURAL CHANGES}

\begin{abstract}
The school is considered a potential space for the development of health care. The Physiotherapist can participate in health actions in the context in question, mainly in adopting postural habits, beginning with the evaluation of postural changes. This study aimed at the postural evaluation of elementary school students in the city of Presidente Prudente. The methodology consisted of the elaboration of a Postural Assessment Sheet containing individual data; questionnaires of body habits and postural attitudes and static postural evaluation. The results were 42 children evaluated; mean age of 9,8 $\pm 0,9$ years; physically active group; good postural perception during daily activities and high prevalence of postural changes, especially in the head, shoulders and lumbar spine segments. We conclude that Elementary School students have good body and posture habits, as well as a high prevalence of postural changes and algias. These aspects reinforce the need for early physiotherapeutic intervention.

Keywords: school health services, physiotherapy, posture, students, elementary school
\end{abstract}

\section{INTRODUÇÃO}

A presença do Fisioterapeuta no ambiente escolar ainda é incipiente na comparação com outros profissionais da Saúde. No entanto, algumas experiências relacionadas à sua atuação com crianças mostram que esse profissional pode contribuir com ações de saúde voltadas para esta população, principalmente no Colloq Vitae 2018 set-dez; 10(3): 21-26. DOI: 10.5747/cv.2018.v10.n3.v239 ISSN 1984-6436/@ 2018 - Publicado pela Universidade do Oeste Paulista. Artigo Open Access sob uma licença CC BY-NC-ND (http://creativecommons.org/licenses/by-nc-nd/4.0/). que diz respeito à postura ${ }^{1,2}$.

Para o Ministério da Saúde a escola é um espaço potencial para o desenvolvimento do cuidado à saúde na promoção de conhecimentos e habilidades para o autocuidado e para a prevenção das atitudes de risco. Esse pensamento resultou na criação do Programa Saúde na Escola (PSE) com a perspectiva de 
ampliar as ações de saúde aos alunos da rede pública de ensino. Um dos componentes estruturais do PSE é a Avaliação das Condições de Saúde e a Elaboração de Estratégias de Promoção e Prevenção que inclui ações relacionadas ao incentivo das práticas corporais e o desenvolvimento de uma cultura de prevenção no ambiente escolar ${ }^{3}$.

No campo da Fisioterapia, ações voltadas para a promoção/prevenção na infância estão bastante relacionadas ao estudo da postura e, geralmente envolvem a elaboração de metodologias de avaliação física para a detecção de alterações e de avaliação do meio escolar na investigação de fatores de risco de posturas inadequadas e, em menor escala, a elaboração de programas de Educação Postural.

Em relação à avaliação postural e do meio escolar parte-se das premissas de que a boa postura resulta de bom controle postural e reflete o estado de equilíbrio de ossos e músculos, para proteção das demais estruturas do corpo humano ${ }^{4}$ e que no período escolar infantil, o sistema musculoesquelético encontra-se em processo de maturação e o uso de mobiliário inadequado, o transporte de cargas excessivas e a manutenção prolongada de posturas estáticas são fatores de risco para o adequado desenvolvimento corporal podendo levar a dores e incapacidades que repercutem negativamente na vida da criança ${ }^{5}$.

Considera-se que a realização de exames posturais simples pode contribuir para 0 diagnóstico de deformidades durante 0 crescimento assim como a investigação do ambiente escolar, como o peso da mochila e o mobiliário escolar que são fatores de risco para as alterações posturais é elemento importante ${ }^{6,7}$. Além disso, esse tipo de avaliação pode possibilitar uma intervenção precoce no próprio ambiente escolar com a elaboração de propostas de Educação Postural que visam a promoção de hábitos posturais saudáveis.

O objetivo deste estudo foi o de avaliar a postura de escolares em uma escola de Ensino Fundamental do município de Presidente Prudente.

\section{MÉTODOS}

Estudo descritivo transversal realizado com 42 alunos dos 4 o e 5 o anos de escola de ensino fundamental do município de Presidente Prudente-SP que frequentavam o Programa
Cidadescola no período vespertino. Todos os alunos avaliados estavam aptos fisicamente a participar do estudo. Todos os pais foram previamente informados da natureza da pesquisa (em palestra proferida na própria escola) e por meio de bilhete informativo. Só participaram do estudo as crianças cujos pais/responsáveis se declararam cientes do estudo e consentiram na participação por meio de TCLE. Este estudo foi aprovado sob o № CAAE 41034914.1.0000.5402.

A avaliação postural foi realizada individualmente com a criança vestida com roupas adequadas (short para meninos e blusa e short para as meninas) em sala disponibilizada pela Escola por meio de Ficha de Avaliação Postural estruturada da seguinte maneira:

a) Identificação de dados pessoais: nome, data de nascimento, idade (anos completos), sexo, ano escolar, nome do responsável e grau de parentesco com o responsável;

b) Identificação antropométrica: peso (em kg) e altura (em metros) e peso da mochila escolar usada pela criança $(\mathrm{em} \mathrm{kg}$ ), medidos por balança digital com estadiômetro da marca Balmak;

c) Questionário semiestruturado de prática de atividade física com itens como: frequência de prática atividade física semanal; tipo de calçado usado para a prática; meio de transporte usado para locomoção até a escola; tempo despendido (em horas diárias) com tecnologias de entretenimento/informação (videogame, televisão, computador/notebook; telefone celular);

d) Questionário estruturado de identificação de aspectos relacionados à postura com itens abordando: posição preferida para dormir; ocorrência de dor nas costas; impacto da dor nas costas em atividades diárias; identificação da intensidade da dor por meio de Escala Visual Analógica com pontuação de zero (sem dor) a 10 (dor máxima).

e) Questionário estruturado de atitudes posturais adotadas no ambiente escolar (indicadas por meio de figuras) abordando três itens (autoidentificados pela criança): o modo que se senta na cadeira da escola ao escrever sobre a carteira; o tipo de mochila usado para carregar o material escolar; o modo de carregar a mochila.

f) Avaliação postural estática

$\mathrm{Na}$ avaliação a criança foi posicionada contra uma parede da sala com marcações em três vistas em relação ao avaliador: Anterior (de frente); Lateral (de lado) e Posterior (de costas). 
$\mathrm{Na}$ vista Anterior foram observados os segmentos cabeça (alinhada, inclinação ou rotação), ombros (simétricos ou elevados), tronco (alinhado ou rotação), crista ilíaca (simétrica ou assimétrica), quadril (normal, rotação interna ou externa) joelhos (alinhados, valgo ou varo).

$\mathrm{Na}$ vista Lateral foram observados: cabeça (normal, projetada para frente ou para trás), ombros (normais, protrusos ou retraídos), coluna cervical (curvatura normal, hiperlordose ou retificação), coluna torácica (normal, hipercifose ou retificação), coluna lombar (normal, hiperlordose ou retificação), cintura pélvica (alinhada, em anteversão ou retroversão), joelhos (alinhados, hiperextensão ou genoflexo) e pés (normais, planos, cavos e varos).
$\mathrm{Na}$ vista Posterior foram observados: ombros (simétricos ou elevados), escápulas (alinhadas, proeminentes, aladas ou retraídas) e coluna vertebral (alinhada ou com escoliose).

Os dados foram tabulados em planilha software Microsoft Office Excel 2007 e apresentados como valores médios \pm desvio padrão, valores absolutos e percentuais.

\section{RESULTADOS}

As características gerais da população podem ser observadas na Tabela 1, em que são apresentados o perfil da população segundo gênero, idade e características antropométricas.

Tabela 1. Características da população estudada (média \pm desvio padrão e variabilidade).

\begin{tabular}{lccc}
\hline \multirow{2}{*}{ Característica } & \multicolumn{3}{c}{ População } \\
\cline { 2 - 4 } & $\begin{array}{c}\text { Crianças } \\
\mathrm{n}=42\end{array}$ & $\begin{array}{c}\text { Meninos } \\
\mathrm{n}=22(52,3 \%)\end{array}$ & $\begin{array}{c}\text { Meninas } \\
\mathrm{n}=20(47,6 \%)\end{array}$ \\
\hline Idade (anos) & $9,8 \pm 0,9(9$ a 12$)$ & $9,8 \pm 0,9(9$ a 12$)$ & $9,8 \pm 0,8(9$ a 11$)$ \\
\hline Peso $(\mathrm{kg})$ & $38,6 \pm 11,1(21,5$ a 65$)$ & $37,0 \pm 9,1(24$ a 55$)$ & $40,4 \pm 12,9(21,5$ a 65$)$ \\
\hline Altura $(\mathrm{m})$ & $1,4 \pm 0,1(1,21$ a 1,65$)$ & $1,4 \pm 0,1(1,29$ a 1,65$)$ & $1,4 \pm 0,1(1,21$ a 1,62$)$ \\
\hline Peso da Mochila $(\mathrm{Kg})$ & $2,8 \pm 1,2(0,5$ a 5$)$ & $2,8 \pm 1,2(0,5$ a 4$)$ & $2,9 \pm 1,2(0,5$ a 5$)$ \\
\hline
\end{tabular}

Em relação aos aspectos relacionados à atividade física observou-se que 36 crianças $(85,7 \%)$ declararam prática regular e sete, $(14,3 \%)$ não regular. Quanto às atividades realizadas foram mencionadas a prática do handebol, basquetebol, voleibol, tênis, futebol, judô, dança e parcour. Em relação ao calçado usado durante o exercício, 28 crianças $(66,7 \%)$ usavam tênis, 10 (23,8\%) faziam descalços e quatro $(9,5 \%)$ usavam chinelo. Quanto ao modo de se deslocar para a escola, 32 crianças $(76,2 \%)$ iam caminhando e 10 (23,8\%) usavam algum meio de transporte.

Na Tabela 2 é mostrado o tempo diário que as crianças despendiam no uso de tecnologias de entretenimento/informação.

Tabela 2. Tempo diário (em horas) despendido no uso de tecnologias de entretenimento/informação

Tempo despendido (em horas)

\begin{tabular}{ccccccc}
\hline Aparelho & 0 a 1 & 2 a 3 & 4 a 5 & 6 ou mais & Não sabe & Não tem \\
\hline $\begin{array}{c}\text { Televisão ou } \\
\text { videogame }\end{array}$ & $13(31,0 \%)$ & $7(16,7 \%)$ & $3(7,1 \%)$ & $6(14,3 \%)$ & $13(31,0 \%)$ & $0(0,0 \%)$ \\
\hline $\begin{array}{c}\text { Computador } \\
\text { ou notebook }\end{array}$ & $3(7,1 \%)$ & $1(2,4 \%)$ & $0(0,0 \%)$ & $1(2,4 \%)$ & $4(9,5 \%)$ & $\begin{array}{c}33 \\
(78,6 \%)\end{array}$ \\
\hline $\begin{array}{c}\text { Telefone } \\
\text { Celular }\end{array}$ & $13(31,0 \%)$ & $7(16,7 \%)$ & $3(7,1 \%$ & $5(11,9 \%)$ & $6(14,3 \%)$ & 8 \\
& & & & & $(19,0 \%)$ \\
\hline
\end{tabular}

Nos aspectos relacionados às posturais no item posição preferida para dormir, 20 crianças $(47,6 \%)$ relataram dormir de lado, 12 $(28,6 \%)$ dormir de barriga para baixo, quatro 
$(9,5 \%)$ de barriga para cima e seis $(14,3 \%)$ não souberam responder.

Em relação à experiência de dor nas costas, 14 crianças $(33,3 \%)$ relataram dor e 28 $(66,7 \%)$ não. Das que sentiram dor, quatro (ou $28,6 \%$ da amostra) afirmaram que a dor limitou a realização de atividades como brincar, estudar ou praticar esportes. Já em relação à intensidade da dor, das 14 crianças, quatro relataram dor leve $(28,6 \%)$, seis $(42,8 \%)$ moderada e quatro $(28,6 \%)$ intensa.

Quanto às questões relacionadas às atitudes posturais na escola observou-se que no item "modo que se senta na cadeira da escola ao escrever sobre a carteira", 14 (33,3\%) apontaram a figura da postura correta e 26 (66,7\%) apontaram figuras relacionadas à postura incorreta. Duas crianças $(4,8 \%)$ não souberam responder.

No item "tipo de mochila usado para carregar o material escolar" observou-se que 37 crianças $(88,1 \%)$ usavam a mochila convencional de duas alças, quatro $(9,5 \%)$ mochila de rodinha e uma criança $(2,4 \%)$ a bolsa-saco.

Já no item "modo de carregar a mochila", das 38 crianças que carregavam a mochila observou-se que $30(78,9 \%)$ o faziam do modo mais correto (mochila apoiada nas costas e uma alça em cada ombro) enquanto que $8(21,1 \%)$ de modo incorreto (alças em apenas um ombro ou carregando na mão).

$\mathrm{Na}$ avaliação postural estática na vista Anterior observou-se que, para o segmento cabeça, 22 crianças $(52,4 \%)$ tinham alinhamento, nove $(21,4 \%)$ com rotação, oito $(19,0 \%)$ com inclinação e três $(7,1 \%)$ com inclinação associada à rotação. Já para ombros, 20 crianças $(47,6 \%)$ tinham simetria e $22(52,4 \%)$ elevação.

No tronco observou-se que 36 crianças $(85,7 \%)$ tinham alinhamento e seis $(14,3 \%)$ em rotação. Quanto às cristas ilíacas, 28 crianças $(66,6 \%)$ tinham simetria, enquanto 14 (33,3\%) assimetria (elevação de um dos lados). 0 quadril estava normal em 35 crianças $(83,3 \%)$, com rotação interna no lado esquerdo em cinco $(11,9 \%)$ e externa em duas $(4,8 \%)$. Quanto aos joelhos observou-se normalidade em 25 crianças (59,5\%), geno varo em $13(30,95 \%)$ e valgo em quatro.

Na Vista Lateral no segmento cabeça, 27 crianças $(64,3 \%)$ apresentaram posição normal, $14(33,3 \%)$ projeção anterior uma criança $(2,4 \%)$ projeção posterior. Quanto aos ombros, 30
$(71,4 \%)$ apresentaram normalidade e $12(28,6 \%)$ protrusão.

$\mathrm{Na}$ avaliação da coluna vertebral, na cervical, 35 crianças $(71,4 \%)$ com curvatura normal, cinco $(11,9 \%)$ hiperlordose e duas $(4,7 \%)$ a retificação; na torácica, 39 com curvatura normal $(92,8 \%)$, duas $(4,8 \%)$ com retificação e uma $(2,4 \%)$ com hipercifose; na lombar, 21 $(50,0 \%)$ com curvatura normal, $18(42,9 \%)$ com hiperlorsose e três $(7,1 \%)$ com retificação.

Já na cintura pélvica, 24 crianças $(57,1 \%)$ apresentaram normalidade, 17 (40,5\%) anteversão e uma $(2,4 \%)$ retroversão enquanto que os joelhos estavam normais em 33 crianças $(78,6 \%)$, em hiperextensão em oito $(19,0 \%)$ e flexão em uma (2,4\%). Quanto aos pés, estavam normais em 24 crianças $(57,1 \%)$, pé plano em 14 $(33,3 \%)$, cavo e varo em duas cada $(4,8 \%)$.

$\mathrm{Na}$ vista Posterior em relação às escápulas, 29 crianças (69,0\%) tinham normalidade, nove $(21,4 \%)$ proeminentes e em quatro $(9,5 \%)$ aladas. Quanto aos ombros observou-se simetria em 17 crianças $(40,5 \%)$ e elevação em 25 (59,5\%). Já a coluna vertebral estava alinhada em 39 crianças $(92,8 \%)$ enquanto que três $(7,1 \%)$ apresentaram escoliose.

\section{DISCUSSÃO}

Avaliações posturais no ambiente escolar, mesmo através de metodologias simples, são boas estratégias de diagnóstico da saúde postural de crianças e na fundamentação de intervenções baseadas em educação de hábitos posturais saudáveis que, por sua vez têm se mostrado importante para a prevenção de agravos da coluna vertebral na fase adulta ${ }^{8}$.

Neste estudo, a avaliação postural foi caracterizada por duas perspectivas: a identificação dos hábitos corporais saudáveis praticados pelas crianças no seu dia a dia e da avaliação física da postura no seu momento estático.

Em relação aos hábitos posturais saudáveis observou-se uma tendência positiva em aspectos relacionados à sua adoção, refletidos pelo uso de mochilas com peso proporcional médio ( $7,3 \%$ neste estudo) abaixo do limite máximo recomendado de $10 \%{ }^{9}$, além de maior prevalência da prática regular de atividade física; do uso adequado de calçado para a realização da prática; do deslocamento ativo até a escola; do pouco tempo gasto no uso das tecnologias de informação; da posição mais 
correta para dormir; no uso de mochila correta e no modo de carregá-la. Apenas no que se refere à postura sentada adotada durante a aula é que prevaleceu o modo incorreto. Estes resultados indicam que as crianças já têm um conhecimento prévio dos hábitos posturais saudáveis o que pode ser um bom indicativo para a adoção de programas de educação postural que reforcem as boas posturas e a imagem corporal, como já demonstrado na literatura ${ }^{10,11}$.

Quanto aos aspectos posturais físicos este estudo obteve resultados semelhantes a outros estudos no que se refere a uma prevalência importante de dor e de alterações posturais dos vários segmentos corpóreos avaliados principalmente em cabeça, ombros e na coluna lombar, ${ }^{2,12}$. Em relação a estas alterações estudos já identificaram associações com alterações fisiológicas de fundo ósseo e muscular ${ }^{12,13}$ e também com o peso e altura da criança ${ }^{14}$, indicando que as crianças deste estudo podem estar em uma situação de risco postural que, se não forem corrigidos podem acarretar problemas importantes em fases mais adiantadas da vida. ${ }^{15}$

Importante salientar que este estudo apresentou limitações características de um estudo descritivo transversal de metodologia de avaliação simples em que não foram realizadas análises mais aprofundadas de associação entre alterações posturais e atitudes posturais, atributos individuais e antropométricos o que poderia permitir estabelecer causais mais precisas.

No entanto, fica evidente a necessidade de futuros estudos sobre os aspectos posturais de escolares de ensino fundamental dada à relevância do tema para a saúde da criança nessa fase tão importante do desenvolvimento humano.

\section{CONCLUSÃO}

Os resultados indicam que, em escolares do ensino fundamental os hábitos corporais e as boas atitudes posturais estão presentes e existe uma prevalência importante de algias e alterações posturais associadas a riscos posturais de desenvolvimento de problemas posturais em fases posteriores da vida. Esses aspectos reforçam a ideia de que estratégias de intervenção fisioterapêutica precoce são necessárias para a promoção da saúde infantil em ambiente escolar.

\section{CONFLITO DE INTERESSE}

Os autores declaram não haver qualquer potencial conflito de interesse que possa interferir na imparcialidade deste trabalho científico.

\section{REFERÊNCIAS}

1. Badaró AF, Nichelle LFI, Turra P. Investigação da postura corporal de escolares em estudos brasileiros. Fisioter Pesq. 2015;22(2):197-204.

2. Neves MMF, Leite JM. R. S. Avaliação Postural em Crianças do Ensino Fundamental. Rev Bras Ciên Saúde. 2016:20(4):285-92. DOI: https://doi.org/10.4034/RBCS.2016.20.04.04

3. Brasil, Ministério da Saúde. Despacho n.으 12.045 de 07 de junho de 2006. Programa Nacional de Saúde Escolar (PNSE). Diário Oficial da República Federativa do Brasil, 110. 2006 jun.

4. Back CMZ, Lima IAX. Fisioterapia na escola: avaliação postural. Fisioter Bras. 2009;10(2):72-7. DOI: https://doi.org/10.33233/fb.v10i2.1505

5. Penha PJ, João SMA, Casarotto RA, Amino CJA, Penteado DC. Postural assessment of girls between 7 and 10 years of age. Clinics. 2005;60(1):9-16.

DOI:

https://doi.org/10.1590/S1807-

$\underline{59322005000100004}$

6. Sedrez JA, Da Rosa MIZ, Noll M, Medeiros FS, Candotti CT. Fatores de risco associados a alterações posturais estruturais da coluna vertebral em crianças e adolescentes. Rev Paul Pediatr. 2015;33(1):72-81. DOI: https://doi.org/10.1016/i.rpped.2014.11.012

7. Souza ACS, Santos GM. A influência do peso da mochila nas alterações posturais em préadolescentes. Ter Man. 2010;38(8):277-84.

8. Rebolho MCT, Casarotto RA, João SMA. Estratégias para ensino de hábitos posturais em crianças: história em quadrinhos versus experiência prática. Fisioter Pesq. 2009;16(1):4651. DOI: https://doi.org/10.1590/S180929502009000100009

9. Candotti $C T$, Noll M, Roth E. Avaliação do peso e do modo de transporte do material escolar em alunos do ensino fundamental. Rev Paul Pediatr. 
2012;30(1):100-6.

DOI:

https://doi.org/10.1590/S0103-

$\underline{05822012000100015}$

10. Candotti CT, De Lemos APA, Noll M. Escola postural para crianças de 10 a 14 anos inserida no contexto do ensino fundamental. Rev Bras Ciên Mov. 2011;19(2):1-17. DOI: https://doi.org/10.18511/0103-

1716/rbcm.v19n2p33-44

11. Vieira A, TL, Candotti CT, Noll M, Bartz PT. Efeitos de um Programa de Educação Postural para escolares do terceiro ano do Ensino Fundamental de uma escola estadual de Porto Alegre (RS). Fisioter Pesq. 2015;22(3):239-45.

12. Klüsener G, Porto DVG. Fisioterapia na escola: avaliação postural em crianças de 6 a 11 anos. Rev Inspirar. 2014;6(5):6-12.

13. Santos CIS, Cunha ABN, Braga VP, Saad IAB, Ribeiro MAGO, Conti PBM, et al. Ocorrência de desvios posturais em escolares do ensino público fundamental de Jaguariúna, São Paulo. Rev Paulista Pediatria. 2009;27(1):74-80. DOI: https://doi.org/10.1590/S0103-

$\underline{05822009000100012}$

14. Penha PJ, João SMA, Casarotto RA, Amino CJ, Penteado DC. Postural assessment of girls between 7 and 10 years of age. Clinics. 2005;60(1):9-16. DOI: https://doi.org/10.1590/S1807$\underline{59322005000100004}$

15. Martelli RC, Traebert J. Estudo descritivo das alterações posturais da coluna vertebral em escolares de 10 a 16 anos de idade. Tangará-SC, 2004. Rev Bras Epidemiol. 2006;9(1):87-93. DOI: https://doi.org/10.1590/S1415-

790X2006000100011

Recebido para publicação em 16/08/2018

Revisado em 23/08/2018

Aceito em 31/08/2018 